\title{
Induction of Canine Deciduoma in Some Reproductive Stages with the Different Conditions of Corpora Lutea
}

\author{
Koichi NOMURA \\ Laboratory of Veterinary Surgery, College of Agriculture, Osaka Prefecture University, 1-1 Gakuen-cho, Sakai, Osaka 593, Japan
}

(Received 13 March 1996/Accepted 6 November 1996)

ABSTRACT. Bitches were examined to see whether canine deciduoma could be induced at some reproductive stages with the different conditions of corpora lutea by inserting a silk suture into the uterine lumen. The bitches stimulated in the early and middle stages of diestrus or in unilateral pregnancy corresponding to these diestrous stages formed deciduoma at a high induction rate, however, no difference in the strength of decidual reaction between the pregnant and diestrous stages was recognized. On the other hand, no reaction could be seen in bitches in late diestrus, the late stage of unilateral pregnancy or the post partum repair phase in which stromal decidual cells similar to those of the rodentia can be seen. In already implanted uteri, however, no deciduoma was formed in the interplacental areas. Even though the corpora lutea were functional, new additional stimulations were not accepted at the interplacental area in which the uterine horn had already been influenced by fertilized ova. From these results, it was suggested that in the dog as well as the rodentia, the endometrium has to be under the influence of functional corpora lutea in order to form deciduoma. — KEY wORDs: canine deciduoma, corpora lutea, decidual cell, post partum uterus, silk suture.

The decidual response of the endometrium in the rodentia, induced by progesterone-stimulated uteri in the absence of fertilized ova by mechanical means, is termed deciduoma $[7,8,20]$. A similar response has been created in the canine uterus at the stage of diestrus corresponding to the implantational phase by various physical means $[13,15-$ 18]. The resulting histological characteristics were not a proliferation of stromal cells as in the rodentia but glandular proliferation and dilatation and/or cystic endometrial hyperplasia $(\mathrm{CEH})$, which had a resemblance to normal early pregnancy, and we proposed that these artificially induced decidual reactions should be called deciduoma in the dog $[14,16]$.

It has also been reported that canine post partum uteri had such decidual cells as can be seen in the stroma of the rodentia [1].

In this study, to clarify more clearly the dependence of canine deciduoma on the corpora lutea, bitches under various luteal conditions including late diestrous and postpartum repair stages were tested to find the best stage for induction of canine deciduoma, and to determine whether decidual cells in the postpartum uterus will proliferate by stimulation through the insertion of a silk suture into the uterine lumen. Furthermore, whether pregnancy could contribute to intensifing the artificial decidual reactions in the contralateral non-pregnant horn is discussed in comparison with those in the uteri of diestrous stages.

\section{MATERIALS AND METHODS}

Fifty mongrel bitches in estrus were used. They were clinically healthy and 8 to $12 \mathrm{~kg}$ in body weight. The bitches were divided into four major groups: diestrous, unilateral pregnant, bilateral pregnant and post partum groups. The diestrous groups were further divided into three subgroups by the difference in time of inserting the suture: early (Group A), middle (Group B) and late diestrus (Group C). The unilateral pregnant groups also were divided into three subgroups and the bilateral pregnant groups into two subgroups according to the difference in the stage of pregnancy: groups $\mathrm{D}$ to $\mathrm{F}$ were treated by inserting a suture into the non pregnant horn in unilateral pregnancy, and groups $\mathrm{G}$ and $\mathrm{H}$ were treated at the interplacental area in bilateral pregnancy. The post partum repair groups were divided into two subgroups; one is in the early (Group I), and the other is in the middle (Group $\mathrm{J}$ ) repair process. Each group consisted of 5 bitches.

All bitches were checked daily for their sex behavior and vaginal smears were taken. The first day of diestrus (Day 1) was determined by the results of these tests and was used as the starting point for the insertion of the suture [10]. The bitches in groups $\mathrm{A}$ to $\mathrm{C}$ were not permitted to copulate, while the bitches in, groups $\mathrm{D}$ to $\mathrm{J}$ were permitted to copulate freely and entered pregnancy. All bitches were laparotomized under general anesthesia with xylazine hydrochloride and ketamine hydrochloride. Then, a silk suture was inserted into the left uterine lumen and the right horn was left intact as a control. The diestrous groups had the silk suture inserted at day 12 for group A, day 24 for group B and day 40 for group C. The unilateral pregnant groups had the suture inserted at day 12 for group D, day 24 for group $\mathrm{E}$ and day 40 for group $\mathrm{F}$. The bilateral pregnant groups had the suture inserted at day 12 for group $\mathrm{G}$ and day 24 for group $\mathrm{H}$. The post partum groups had the suture inserted at 7 days and 40 days after delivery for groups I and $\mathrm{J}$ respectively. In the groups $\mathrm{D}$ to $\mathrm{F}$, the bitches in their anestrous stage, also received a prior operation of double ligatures to their left ovarian tube, and fundus of the uterine horn at the bifurcation to prevent the fertilized ova transfering to the left horn before the insertion of the suture. 
All bitches were laparotomized again and ovariohysterectomized 12 days after inserting the suture. The ovary and uterus were observed grossly and fixed with $10 \%$ buffered formalin solution.

Microscopical sections were prepared from these organs by routine methods and stained with Hematoxylin and Eosin. The histological evaluation of deciduoma was performed to determine whether the proliferation and dilatation of the uterine glands and/or cystic endometrial hyperplasia (socalled "Swiss cheese endometrium" according to Bloom [5]) could or could not be seen.

\section{RESULTS}

Incidence of deciduoma: The incidence of deciduoma is shown in Table 1. The highest rate of incidence (100\%) was observed in the phase of early diestrus (group A), and in early and middle unilateral pregnancy (groups D and E). However, in the groups which had a suture inserted into the interplacental area (groups $\mathrm{G}$ and $\mathrm{H}$ ), deciduoma could not be induced. The post partum phase (groups I and J) also produced no deciduoma.

Macroscopical observation of the uterus: The left uterine horn of all bitches in which deciduoma could be induced was enlarged and rosary-shaped (groups A, B and D, E). The endometrium was obviously hyperplastic and appeared to have many thick folds accompanied with many small pleomorphic vesicles on it. In some of these cases, a small amount of jelly-like material adhered to the surface of the endometrium. The magnitude or strength of these left uterine changes were not different between the pregnant and diestrous groups (Figs. 1 and 2). In the late pregnancy group (group F), however, the left uterine horn had no deciduoma but did have slight hyperplasia of the uterine
Table 1. Incidence of canine deciduoma at various conditions of corpora lutea

\begin{tabular}{|c|c|c|}
\hline Groups & $\begin{array}{c}\text { Insertion } \\
\text { of Silk Suture }\end{array}$ & $\begin{array}{c}\text { Incidence } \\
\text { of Deciduoma }{ }^{\text {b) }}\end{array}$ \\
\hline \multicolumn{3}{|l|}{ Diestrous groups } \\
\hline A. early diestrus & 12 & $5 / 5$ \\
\hline B. middle diestrus & 24 & $4 / 5$ \\
\hline C. late diestrus & 40 & $0 / 5$ \\
\hline \multicolumn{3}{|l|}{ Unilateral pregnant groups } \\
\hline D. early pregnancy & 12 & $5 / 5$ \\
\hline E. middle pregnancy & 24 & $5 / 5$ \\
\hline F. late pregnancy & 40 & $0 / 5$ \\
\hline \multicolumn{3}{|l|}{ Bilateral pregnant groups } \\
\hline G. early pregnancy & 12 & $0 / 5$ \\
\hline H. middle pregnancy & 24 & $0 / 5$ \\
\hline \multicolumn{3}{|l|}{ Post partum groups } \\
\hline I. early post partum & p. p. 7 & $0 / 5$ \\
\hline J. middle post partum & p. p. 40 & $0 / 5$ \\
\hline
\end{tabular}

a) Day 1 is the first day of diestrus, p.p.: Days after delivery. b) Positive cases/total cases.

wall. In the bilateral pregnancy groups (groups $\mathrm{G}$ and $\mathrm{H}$ ), each left horn also had no deciduoma, but showed some large ampoule-like shapes in which some aborted fetuses were included (Fig. 3).

In the post partum groups (groups I and J), no deciduoma was observed in the uteri of any of the bitches. In group I, some coagulated, creamy pseudomembranous bands were seen on the maternal placental areas in both the right and left horns. In addition, there was dark brown lochia in the left horn (Fig. 4). In the bitches of group J, both horns no longer had segmental annular rings (which had been formed of placenta) on the surface of the uterus, and some brown or black colored thin bands were recognized on the

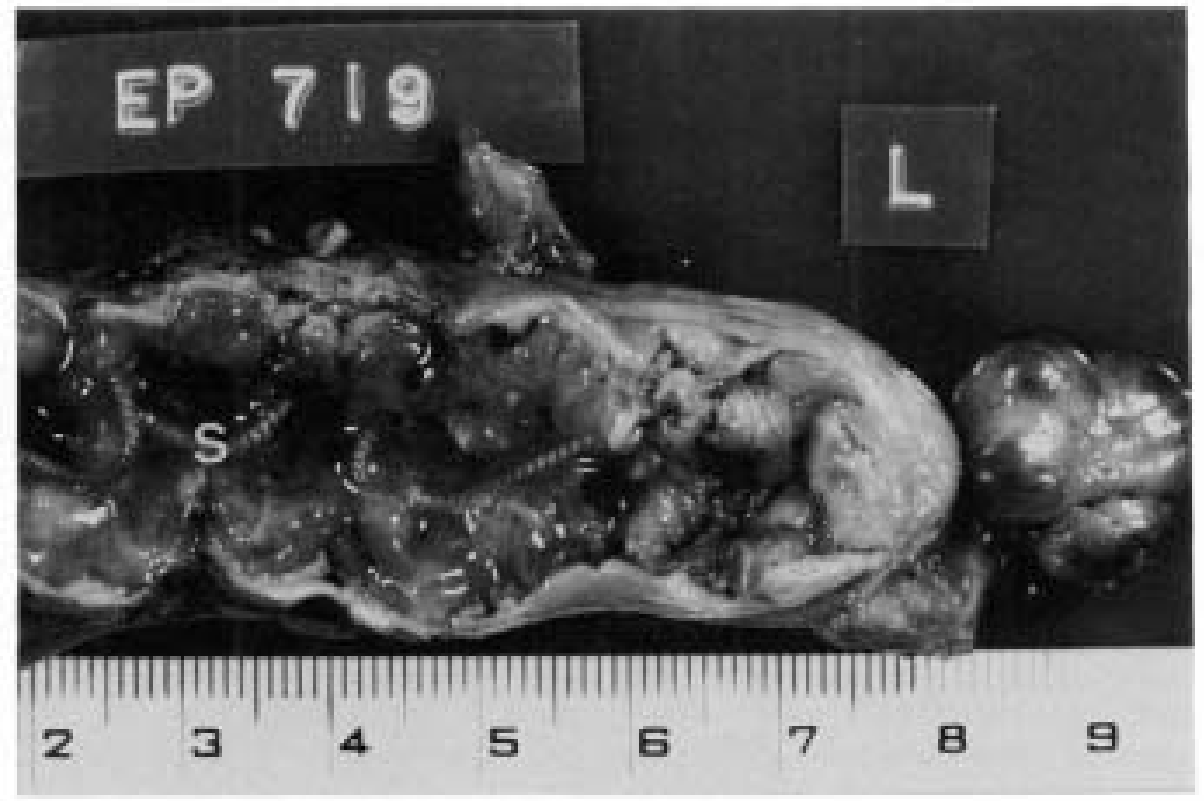

Fig. 1. The left uterine horn in middle diestrus (group B) showed a marked endometrial hyperplasia induced by inserting a suture (S). 


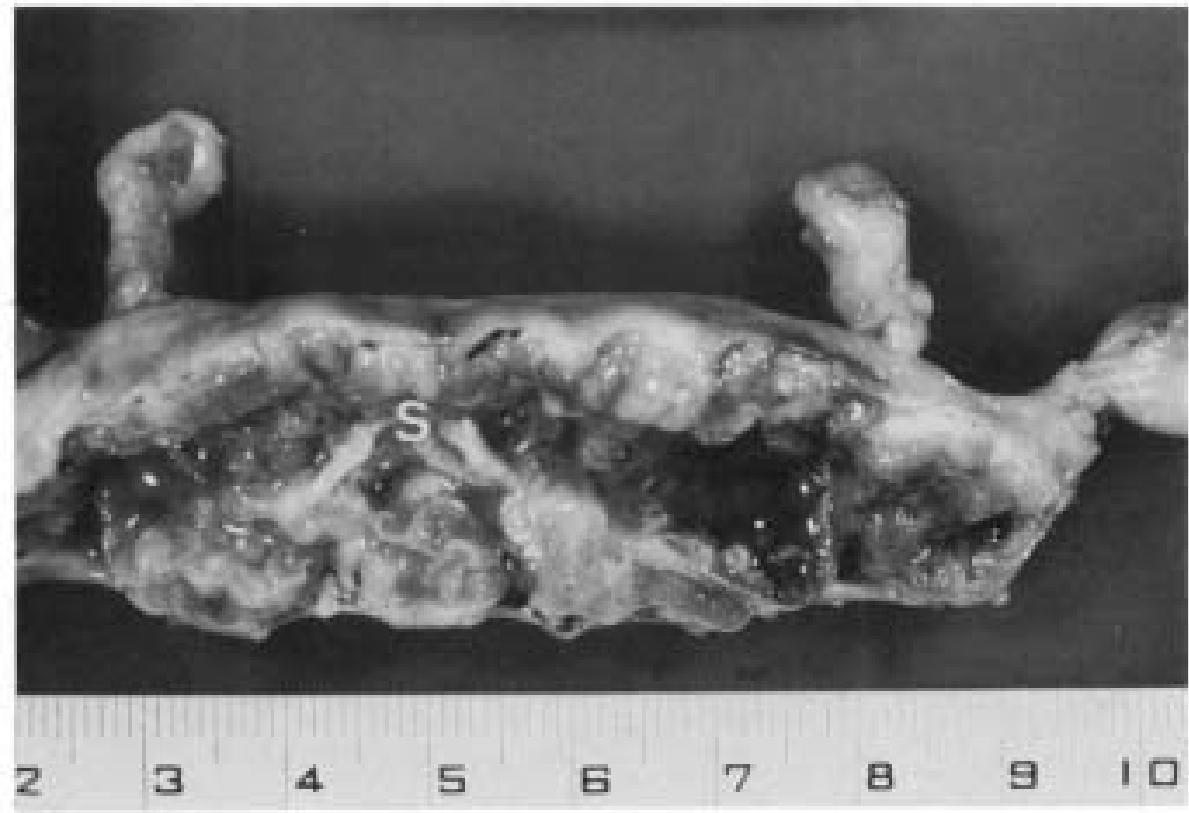

Fig. 2. The left non-pregnant uterine horn, stimulated by inserting a suture in unilateral middle pregnancy (group E), showed almost the same magnitude of endometrial hyperplasia as that in the diestrous group (Fig. 1).

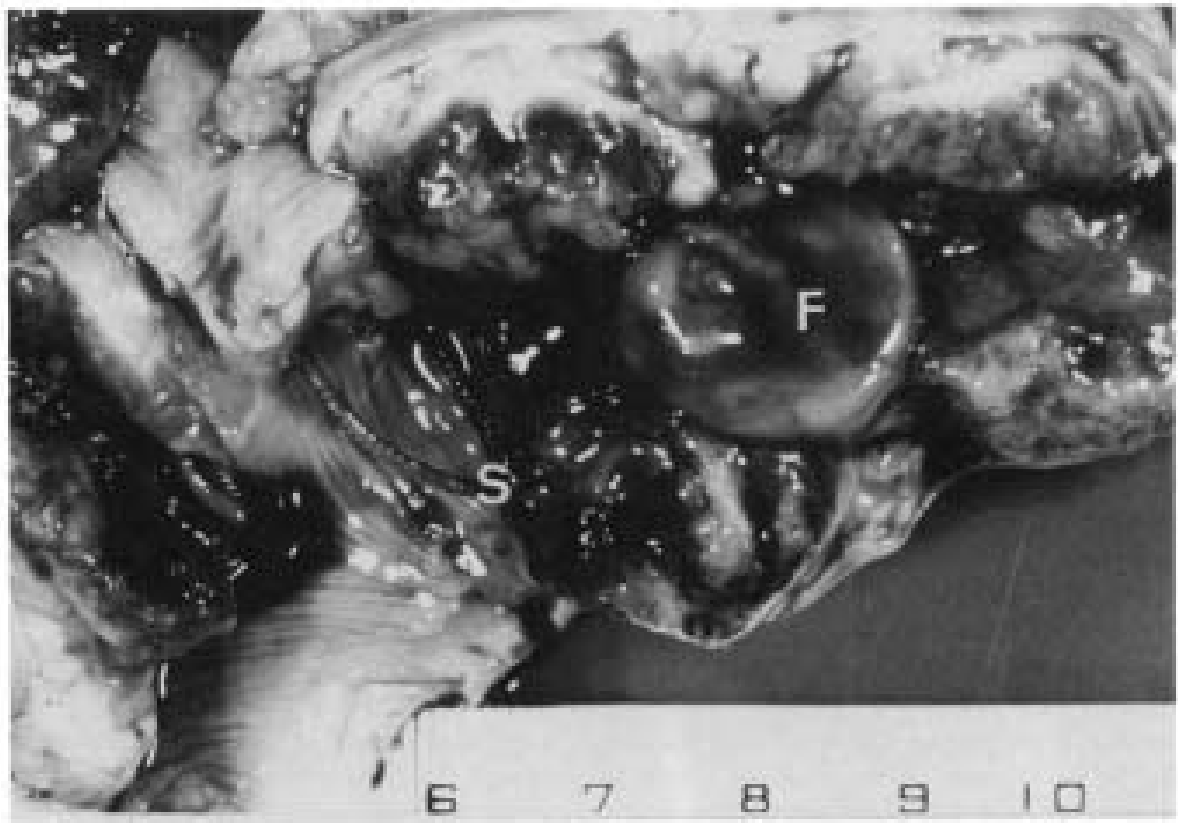

Fig. 3. The left horn into which a suture was inserted in the interplacental area in bilateral middle pregnancy (group H), not only formed a deciduoma but also induced abortion in the same horn. The opposite horn, with no stimulation, sustained the pregnancy. S: suture, F: aborted fetus.

endometrium in the left uterine horn, which showed the traces of maternal placenta.

Macroscopical observation of the ovaries: The ovaries appeared macroscopically normal, i.e., the ovaries in the cases of groups A to $\mathrm{H}$ contained one or more corpora lutea in various stages corresponding to the days after estrus. In post partum bitches (groups I and J), the ovaries mainly contained some degenerating corpora lutea.

Histological findings of the uterus: Almost all the bitches which had a deciduoma at the site of suture insertion showed 


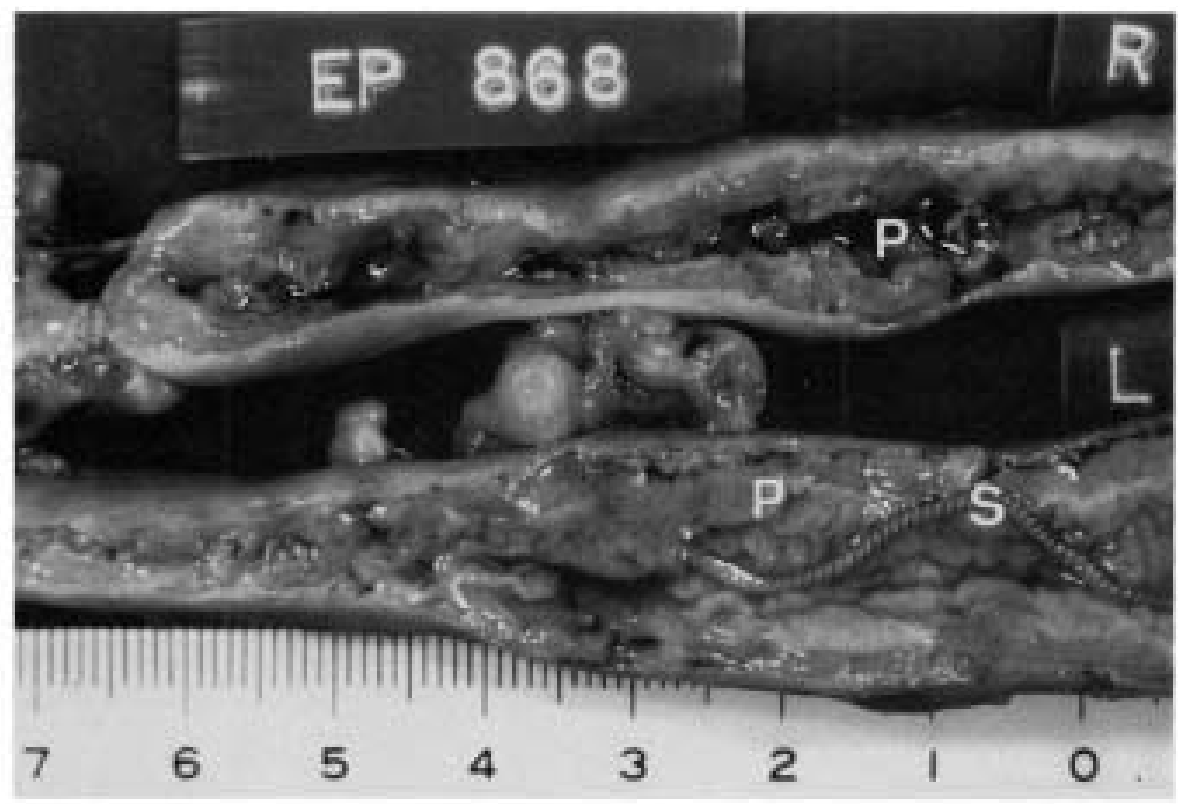

Fig. 4. Both uterine horn in the early post partum (group I) are shown. Almost all lochia is removed from left uterine lumen. The left uterine horn into which a suture was inserted showed a little hypertrophy. The right horn had the appearance of a normal post partum uterus. S: inserted suture, $P$ : remaining tissues of the degenerated maternal placental area.

a marked proliferation and dilatation of the uterine glands (groups A, B, D and E). These changes showed highly irregular patterns with cystic or reticular arrangements compared to those of the control uterine endometrium. In other words, these uterine structures resembled histologically the so called "Swiss cheese endometrium". These proliferated uterine glands showed a high secretory activity, and vesicle formation in some glands could also be seen.

In the endometrium where deciduoma did not develop, in spite of the insertion of a suture, there was no hyperplasia of the glands, but a mild inflammatory tissue zone was recognized in the stroma of the subepithelial area (groups B and $\mathrm{C}$ ). In other cases detachment of epithelial cells and fibrillation of the superficial layer were sometimes observed, as well as edema and hemorrhage in the stroma of the affected areas. In addition, compared with the control endometrium in the right uteri (no suture site), the affected areas showed slightly more differentiation of the uterine glands, but none developed into cystic dilatation.

In all cases of the post partum phase (groups $\mathrm{J}$ and $\mathrm{K}$ ), no $\mathrm{CEH}$ was induced. In these cases, almost all the normal histological structures of the post partum endometrium were observed except for a slight edema or hemorrhage in the stroma and cellular infiltration of small round cells, or histiocytic cells, in the superficial layer of the endometrium adhering to the suture in the left uterus. These post partum decidua gradually degenerated into fibrinoid tissue at the upper part of the endometrium and covered the underlying tissue including the basal glandular layer (Fig. 5). In the stroma of the post partum decidua, decidual cells could be found in these infiltrated cells. Especially, many decidual cells appeared to gather on the border between the decidua and basal glandular layer (Fig. 6.).

\section{DISCUSSION}

The induction rate of canine deciduoma kept a high level until the middle diestrus (Day 24), and then the rate dropped suddenly in the late diestrus (Day 40). This result indicates the possibility that uterine responses change drastically with differences in the time for stimulation. It has been reported that the level of blood progesterone is gradually increases from the first day of diestrus and reaches a peak value near the middle of diestrus, after which it gradually decreases towards the end of diestrus [6,9]. It could therefore be suggested that the drastic changes on the uterus, seen in this study, might be closely related to the rise and fall of blood progesterone levels in diestrus.

In this study, the relationship between the pregnant and diestrous stages on the induction rate and magnitude of deciduoma was also examined and the results showed no remarkable differences between them, as shown in the Table 1. That is, in spite of non-pregnancy, the diestrous stages showed the same high-induction rate and strength of magnitude of deciduoma as those of unilateral pregnancy. This suggests that canine deciduoma received no effects from the pregnancy in the opposite side, and that the sustaining of pregnancy had no influences from the deciduoma induced in the opposite side horn. The uterus in diestrus may also possess the same potential for reaction to stimulation as to pregnancy, which is consistent with reports that the levels of blood progesterone in diestrus were not so 


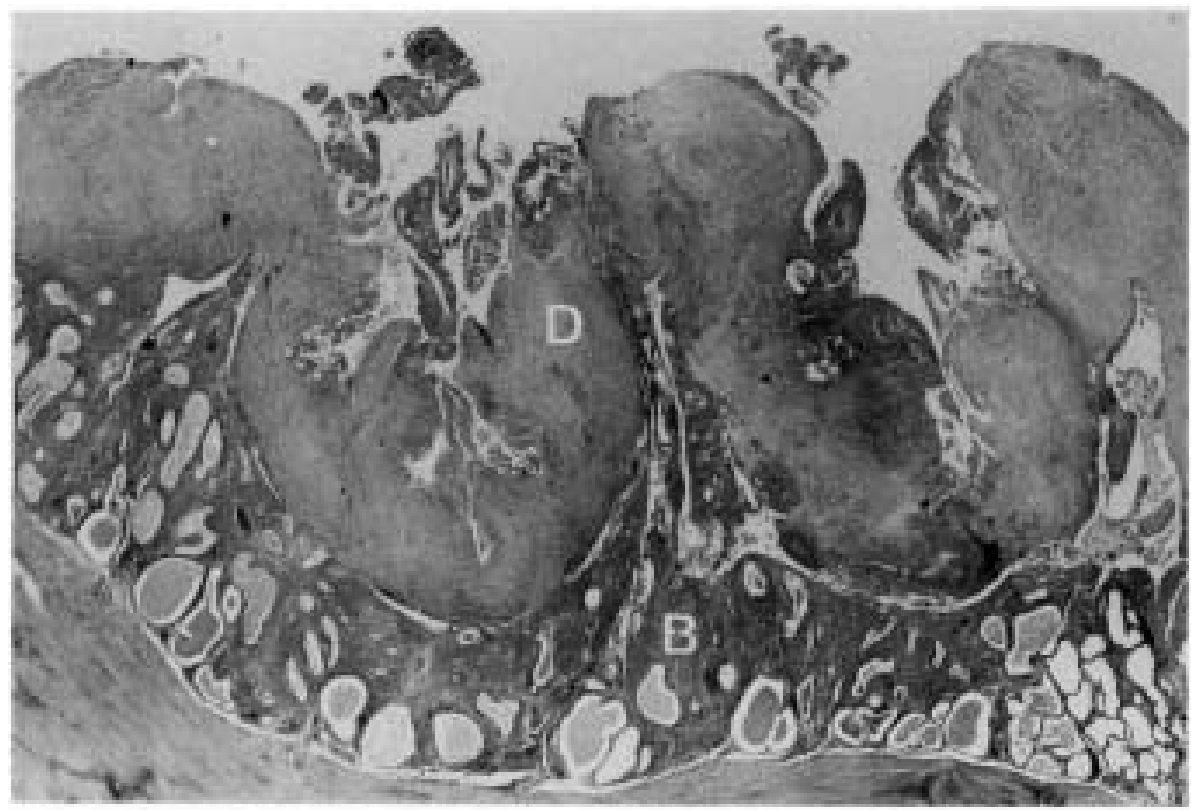

Fig. 5. The histological section from Fig. 4. Decidua in the upper part of maternal placenta degenerated into fibrinoid tissue. This degenerated tissue zone was gradually torn off from the basal glandular layer. D: decidua, B: basal glandular layer. $\mathrm{H} \bullet \mathrm{E}$ stain.

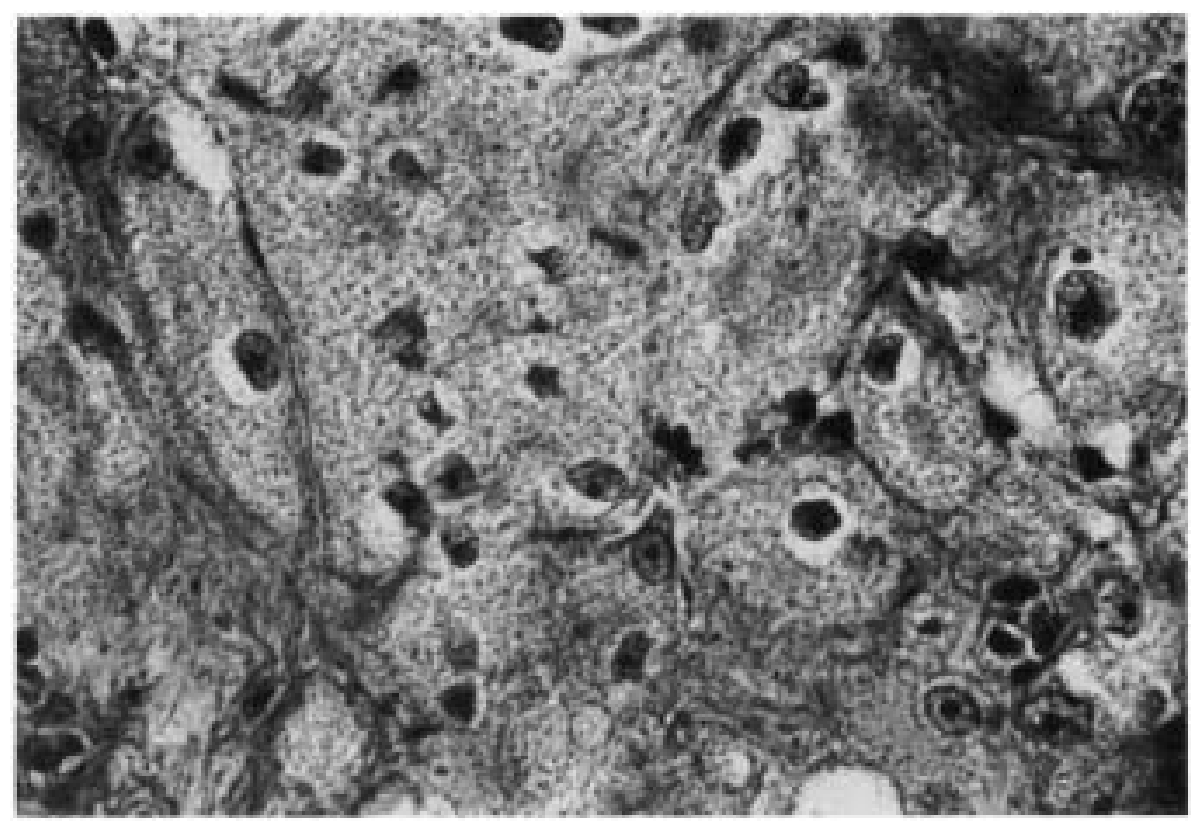

Fig. 6. A histological section was taken from the bottom of the degenerated decidua shown in the Fig. 5. There are many decidual cells, which have a round or an oval shape with a large foamy cytoplasm group in that area. Accumulation of these cells was not a reaction resulting from the suture insertion but also a normal post partum physiological reaction. These cells gradually fall into fat necrosis and then form large vacuoles. Finally, degenerated decidua is torn off from a line of these vacuolated zone. $\mathrm{H} \bullet \mathrm{E}$ stain. 
much different from those in pregnancy $[12,19]$.

In the other gravid uteri which were stimulated by inserting a suture into the interplacental areas (groups G and $\mathrm{H}$ ), not only could deciduoma not be induced but also embryos were aborted in that side. It was supposed that new additional stimuli were not accepted when any decidual reactions (e. g. normal pregnancy) had already been progressing at other parts in the same horn. Moreover, the results also suggest that the new stimulus induced so strong an eliminating action that abortion of the embryos was also caused.

It is well known that many deciduata have so-called decidual cells. In the rodentia, these cells already appear in the uterine stroma at the implantational phase and gradually proliferate according to the progress of pregnancy. These cells have abundant glycoprotein in their cytoplasm and are attributed to nutrition for early embryos $[2,7,11]$. On the other hand, while the dog has a decidua, canine embryonic nutrition does not depend on decidual cells, but on histotrophe originating from the proliferated and dilated uterine glands (uterine milk) [3, 4]. AL-Bassam reported that the decidual cells originated from the stroma like those of the rodentia appear in the canine post partum uterus [1].

This report was our inspiration to examine whether canine decidual cells are responsive to stimulation and could proliferate as well as the rodentia stroma cells do. The results of this study demonstrate that the canine decidual cells did not proliferate and deciduoma could not be induced, and at the end of the experiments, these decidual cells histologically showed fatty degeneration and formed a foamy appearance with many small or large vacuoles in cytoplasm. It is suggested that these cells already had no ability to proliferate at the time of stimulation and gradually began fatty degeneration during the experimental period.

In conclusion, in this study, it has been shown that the dog deciduoma could not be induced even if stimulation was added to the stromal decidual cells which could be observed in the post partum uterus (Fig. 6). Moreover, scince canine deciduoma could be easily induced during the functional luteal stage, from early to middle diestrus, but with difficulty during the regressive stage of corpora lutea in late diestrus, it is suggested that canine deciduoma might also be dependent on functional corpora lutea like the rodentia. Further studies will, however, be needed to confirm this hypothesis.

\section{REFERENCES}

1. AL-Bassam, M. A., Thomson, R. G., and O'Donnell, L. 1981. Normal postpartum involution of the uterus in the dog. Can. J. Comp. Med. 45: 217-232.

2. Amoroso, E. C. 1952. The mammalian placenta. pp. 138159. In: Marshall's Physiology of Reproduction, vol. 2, 3rd ed. (Parkes, A. S. ed.), Longmans Green and Co., London, New York, Toronto.

3. Amoroso, E. C. 1952. Placenta endotheliochorialis. pp. 211-
242. In: Marshall's Physiology of Reproduction, vol. 2, 3rd ed. (Parkes, A. S. ed.), Longmans Green and Co., London, New York, Toronto.

4. Barrau, M. D., Abel, J. H. , Verhage, H. G., and Tietz, W. J. 1975. Development of the endometrium during the estrous cycle in the bitch. Am. J. Anat. 142: 47-66.

5. Bloom, F. 1954. Hyperplasia of the endometrium. pp. 344 349. In: Pathology of the Dog and Cat. The Genitourinary System, with Clinical Considerations. Am. Vet. Pub. Inc., Evanston, Illinois.

6. Christie, D. W., Bell, C. E., and Palmer, R. F. 1971. Peripheral plasma progesterone levels during the canine oestrous cycle. Acta Endocrinol. 68: 543-550.

7. De Feo, V. J. 1967. Decidualization. pp. 191-276. In: Cellular Biology of the Uterus (Wynn, R. M. ed.), Appletone, Century, Crofts.

8. Finn, C. A. and Porter, D. G. 1975. The decidual cell reaction. pp. 74-84. In: The Uterus (Finn, C. A. and Porter, D. G. eds.), Elek Science, London.

9. Hadley, J. C. 1975.Total unconjugated oestrogen and progesterone concentrations in peripheral blood during the oestrous cycle of the dog. J. Reprod. Fertil. 44: 445-451.

10. Holst, P. A. and Phemister, R. D. 1974. Onset of diestrus in the beagle bitch. Definition and significance. J. Vet. Res. 35: 401-406.

11. Mossman, H. W. 1937. Comparative morphogenesis of the fetal membranes and accessory uterine structures. Contrib. Embryol. Carneg. Instn. 26: 129-246.

12. Nett, T. M., Akabar, R. D., Phemister, P. A., Holst, P. A., Reichert, J. R., and Niswender, G. D. 1975. Levels of luteinizing hormone, estradiol and progesterone in serum during the estrous cycle and pregnancy in the beagle bitch. Proc. Soc. Exp. Biol. Med. 148: 134-139.

13. Nomura, K., Kawasoe, K., and Shimada, Y. 1990. Histological observations of canine cystic endometrial hyperplasia induced by intrauterine scratching. Jpn. J. Vet. Sci. 52: 979983.

14. Nomura, K. 1994. Induction of a deciduoma in the dog. $J$. Vet. Med. Sci. 56: 365-369.

15. Nomura, K. 1995. Histological evaluation of canine deciduoma induced by silk suture. J. Vet. Med. Sci. 57: 9-16.

16. Nomura, K. 1995. Histological evaluation of canine decidual reactions induced by intraluminal injection of olive oil. $J$. Vet. Med. Sci. 57: 71-74.

17. Nomura, K. 1996. Radiographical and histological evaluation of canine decidual reaction induced by intraluminal injection of bouillon solution mixed with or without barium sulfate. $J$. Vet. Med. Sci. 58: 145-149.

18. Nomura, K. 1996. Canine deciduoma induced by intraluminal insertion of uterine grafts. J. Vet. Med. Sci. 58: 151-155.

19. Smith, M. S. and Mcdonald, L. E. 1974. Serum levels of luteinizing hormone and progesterone during the estrous cycle, pseudopregnancy and pregnancy in the dog. Endocrinology 94: 404-412.

20. Stedman's Medical Dictionary. 1972. 22nd ed. p. 325. “deciduoma" The Williams \& Wilkins Co., Baltimore.

21. Steven, D. 1975. Anatomy of the placental barrier. pp. 25 57. In: Comparative Placentation Essays in Structure and Function (Steven, D. H. ed.), American Press, London, New York, San Francisco. 